\title{
Rationale, design and methodology for a Prospective Randomized Study of graft patency in Off-pump and On-pump MultI-Vessel coronary artery bypasS Surgery (PROMISS) using multidetector computed tomography
}

\author{
Miguel Sousa Uva*†1, Fernando Matias ${ }^{1}$, Sara Cavaco ${ }^{\dagger 2}$ and \\ Manuel Pedro Magalhães ${ }^{\dagger 1}$
}

Address: ${ }^{1}$ Departamento da Circulação, Serviço de Cirurgia Cardíaca, Hospital da Cruz Vermelha, Lisboa, 1549-008, Portugal and ${ }^{2}$ Division of Behavioural Neurology and Cognitive Neuroscience, University of Iowa, Iowa City, USA

Email: Miguel Sousa Uva* - uvamiguel@hotmail.com; Fernando Matias - fernando.matias@hcvp.com.pt; Sara Cavaco - sara-cavaco@uiowa.edu; Manuel Pedro Magalhães - pedro.magalhaes@hcvp.com.pt

* Corresponding author †Equal contributors

Published: 17 July 2008

Trials 2008, 9:44 doi:10.1/86/1745-62/5-9-44

This article is available from: http://www.trialsjournal.com/content/9/I/44

(c) 2008 Uva et al; licensee BioMed Central Ltd.

This is an Open Access article distributed under the terms of the Creative Commons Attribution License (http://creativecommons.org/licenses/by/2.0), which permits unrestricted use, distribution, and reproduction in any medium, provided the original work is properly cited.
Received: 6 May 2008

Accepted: 17 July 2008

\begin{abstract}
Background: Off-pump coronary artery bypass grafting has been accused of possibly compromising graft patency. Sixteen slice computed tomography has shown good diagnostic accuracy in the assessment of coronary bypass graft patency when compared with conventional coronary artery angiography and is less invasive. The study hypothesis is that coronary artery bypass grafting (CABG) performed without cardiopulmonary bypass (Off-Pump) has equivalent early graft patency as if performed with cardiopulmonary bypass (On-Pump) and may have reduced complication rate.
\end{abstract}

Methods/Design: The Prospective Randomized Comparison of Off-Pump and On-Pump Multlvessel Coronary Artery BypasS Surgery (PROMISS) is a controlled, single blinded, single centre clinical trial, comparing early graft patency using I6-slice computed tomography in patients with multi-vessel coronary artery disease operated either without or with extracorporeal circulation. Inclusion criteria are multivessel disease with an indication for first time, isolated, non emergent coronary artery bypass grafting with a minimum of three distal anastomoses. Secondary end points are peri-operative mortality, combined morbidity, length of stay, neuro-cognitive testing at 6 weeks and adverse events, stress test and quality of life at 6 months and one year. The sample size of one hundred and fifty patients was calculated in order to enable the detection of a $5 \%$ difference in graft patency, with $80 \%$ power, considering a minimum of 3 distal anastomoses per patient. Enrolment started in April 2005 and ended July 2007 with study closure in July 2008.

Conclusion: The PROMISS trial aims to shed new light on the effect of Off-Pump as compared to On-Pump coronary artery bypass surgery on graft patency, assessed by multidetector computed tomography, in unselected patients with multivessel coronary artery disease.

Trial Registration: Current Controlled Trials ISRCTN58800729 


\section{Background}

Coronary artery bypass surgery is a proven and effective mode of treatment for ischaemic heart disease. It provides relief of symptoms and increases survival, in particular in patients with left main disease and two or three vessel disease including the proximal left anterior descending artery [1].

Coronary artery bypass grafting (CABG) can be performed in several ways but in the majority of cases it is still performed with the assistance of cardiopulmonary bypass (CPB), aortic cross clamping and cardioplegia induced cardiac arrest ("on-pump" or conventional method). Coronary artery bypass grating can also be performed without CPB ("off-pump" or OPCAB) and this method's application varies from around $20 \%$ in the USA and Europe to more than 50\% in India and Japan.

As a consequence of increased life expectancy and a more widespread use of coronary angiography with exponential growth of percutaneous coronary interventions in ischaemic heart disease, patients proposed for CABG are older and suffer from more co-morbid conditions. In spite of this, continuous incremental improvements in peri-operative patient management, anaesthetic and surgical techniques have led to better results [2]. However, morbidity is still significant in certain patient subsets and off-pump coronary artery bypass surgery saw its development in the nineties with the rationale of reducing some of the cardiopulmonary bypass related complications [3]. Indeed, although CPB is usually well tolerated in the majority of cases, it can be responsible for complications and death, particularly in older and sicker patients with co-morbid conditions. Cardiopulmonary bypass may be a source of distal embolization by aortic canulation and manipulation and causes myocardial ischaemia-reperfusion injury through cardioplegic arrest. CPB causes also a systemic inflammatory response and increases the need for blood transfusion by hemodilution and coagulation disturbances [4].

Most studies show that OPCAB is associated with less cardiac, pulmonary, renal or neurologic complications and reduces blood transfusion and hospital stay compared with "on-pump" coronary bypass surgery [5-7]. However, there is still some debate about the type and degree of morbidity reduction achieved by OPCAB [8]. For example, neurocognitive decline is one of the most frequent sequelae of cardiac surgery but there is still no consensus whether OPCAB is superior to conventional CABG with respect to this complication [8]. Neuropsychological evaluation is based on a battery of tests evaluating memory, learning, attention, psychomotor speed, dexterity and diverging results between studies, may be due to different assessment protocols, different timings, statistical tools used to define a decline and absence of control groups.

In general, there has been difficulty in accumulating scientific evidence for OPCAB benefit, due to low incidence of events (mortality, stroke, myocardial infarction), therefore requiring very large samples or high risk populations to demonstrate a statistically significant improvement [9].

On the other hand, "off-pump" CABG has been accused of compromising completeness of revascularisation and graft patency by its increased technical difficulty, particularly in grafting the left ventricular lateral wall [10]. Indeed, a learning curve applies in OPCAB, and its benefits can be expected only with the strict application of principles and rules and a sustained practice without which results can be inferior to those obtained with conventional "on-pump" CABG. The same applies to long term results which depend on completeness and quality of revascularisation.

Coronary artery bypass grafting has an important role in the treatment of coronary artery disease as shown by series with long term results [11]. However, increased survival and symptoms relief are directly related to graft patency. Consequently, it is important that new, less invasive methods reduce the early risk of the procedure but also ensure the same revascularisation quality as the conventional technique.

The justification for theProspective Randomized Comparison of Off-Pump and On-Pump MultI-vessel Coronary Artery BypasS Surgery (PROMISS) derives from current discordant data in the literature regarding the potential inferior graft patency when CABG is performed off-pump as compared with the arrested heart on-pump procedure [12-15]. There would be no advantage to the patient if a short term benefit was to be compromised by the ultimate goal of achieving perfect myocardial revascularisation. In fact, while some studies show no difference in graft patency, others show decreased graft permeability when $\mathrm{OPCAB}$ is used [12-15].

Many procedural and surgeon related factors (exposure, stabilisation, shunt use, operator's experience and patient selection) can account for these differences so it is important to evaluate both off and on-pump coronary bypass surgery using standardised techniques, unbiased assessment of results and an accurate patency control method.

Until recently, coronary angiography was the only method to assess coronary artery bypass graft patency. This technique is stressful, causes discomfort and has local (limb ischemia, haematoma, false aneurysm), neurological (stroke, TIA) and cardiac (graft dissection, myocardial 
infarction, arrhythmias) complications in up to $1 \%$ of cases [16]. From an ethical standpoint, a potential life threatening complication risk in asymptomatic patients discouraged us from embarking on a randomized study using conventional coronary angiography.

Recent advances in multidetector computed tomography (MDCT) technology, namely with reduced acquisition time and improved spatial resolution, has permitted its application to the detection of coronary artery disease and bypass graft evaluation [17]. Relative graft immobility and freedom from calcification allows multidetector computed tomography to achieve high diagnostic accuracy for coronary bypass graft patency evaluation [18-20]. This led to the design of a randomized but non invasive study of coronary bypass graft patency, comparing on and offpump techniques and the conception of PROMISS.

PROMISS primary objective is to compare early coronary bypass graft patency by 16 slice computed tomography in patients with multivessel coronary artery disease undergoing first time coronary artery bypass grafting with (onpump) and without (off-pump) cardiopulmonary bypass.

Secondary endpoints are:

1- Mortality and morbidity in patients operated with onpump and off-pump.

2- Comparative changes in neuro-cognitive performance between base line and 4-6 weeks by a battery of neuropsychological tests.

3- Evaluation of functional status, ischaemic threshold by stress test, quality of life and adverse events at 6 months and one year.

Finally, as tertiary end point we aim to analyse hospital costs.

\section{Methods}

PROMISS is a prospective, randomized, single blinded, single centre study.

\section{I Organization}

Patient inclusion, surgery, post operative care, multidetector computed tomography and follow-up are conducted at Hospital da Cruz Vermelha Portuguesa. The principal investigator designed the study and is the surgeon responsible for performing all on-pump and off-pump operations. The principal investigator has a large experience in on-pump CABG and has performed more than 300 OPCAB surgeries before initiating the study.

\subsection{Ethical Issues}

PROMISS is registered in The International Society for Clinical Randomized Trials (ISRCTN58800729), it is conducted in accordance with the principles of The Declaration of Helsinki, with the Portuguese laws and rules and subscribes to the principles outlined in the International Conference on Harmonisation of Good Clinical Practice [21].

All patients receive full explanation of study objectives, the operations to be performed, with or without $\mathrm{CPB}$ according to randomization, its risks and benefits and signed the informed consent form.

Any death during the study period requires the hospital ethical commission to be informed.

\subsection{Recruiting Process}

All patients that comply with the inclusion and exclusion criteria are considered for enrolment in PROMISS (Table 1). Inclusion requires consensus between two surgeons regarding the operative plan: number of grafts with at least three distal anastomoses and target vessels to bypass. This operative plan is then recorded in the patient's case report form before the operation.

No patient is excluded because of recent myocardial infarction, ventricular dysfunction, or poor quality target vessels (size, location, calcification). No patient is excluded for associated morbid conditions with the exception of renal insufficiency (because of iodine administration) and atrial fibrillation (multidetector computed tomography imperative).

Table I: Eligibility Criteria

\begin{tabular}{ll}
\hline Inclusion & Exclusion \\
\hline Multivessel CAD & IV inotropes, IABP, Assisted Ventilation \\
First Time CABG with $\geq 3$ grafts & Associated Surgical Procedure \\
Age 30-90 years & Creatinin > I.5 ULN \\
Signed Informed Consent & Chronic Atrial Fibrillation \\
& Iodine Allergy \\
& Non Menopaused Woman \\
& Inability to Give Informed Consent
\end{tabular}


During the study period, a patient diagram flow is used indicating patients screened, patients enrolled and reasons for exclusion, patients randomized and patients available for MDCT and follow up according to the CONSORT recommendations (Figure 1) [22].

\subsection{Randomization}

The informed consent is signed before randomization. Randomization is performed by the method of random permuted blocks. A sealed envelope containing the patient's number is opened in the operating room before the beginning of the operation.

\section{5 "Cross-over" and study discontinuation}

Cross-over from one group to another is possible in case patient safety is deemed at risk, a condition that is estimated to occur in less than $5 \%$ of the cases:
- From on-pump to off-pump group in case of calcified ascending aorta.

- From the off-pump to the on-pump group in case of refractory hemodynamic deterioration.

Patients who cross over from off-pump to on-pump are analysed in the original off-pump group for which he or she was randomized in intent to treat analysis.

Premature exit from the study is possible at any moment at patient's will or because of inclusion/exclusion criteria violation.

\subsection{Surgical technique and post operative care}

Premedication, anaesthetic protocol, patient opening and closing, harvesting and graft anastomoses techniques are the same in both groups.

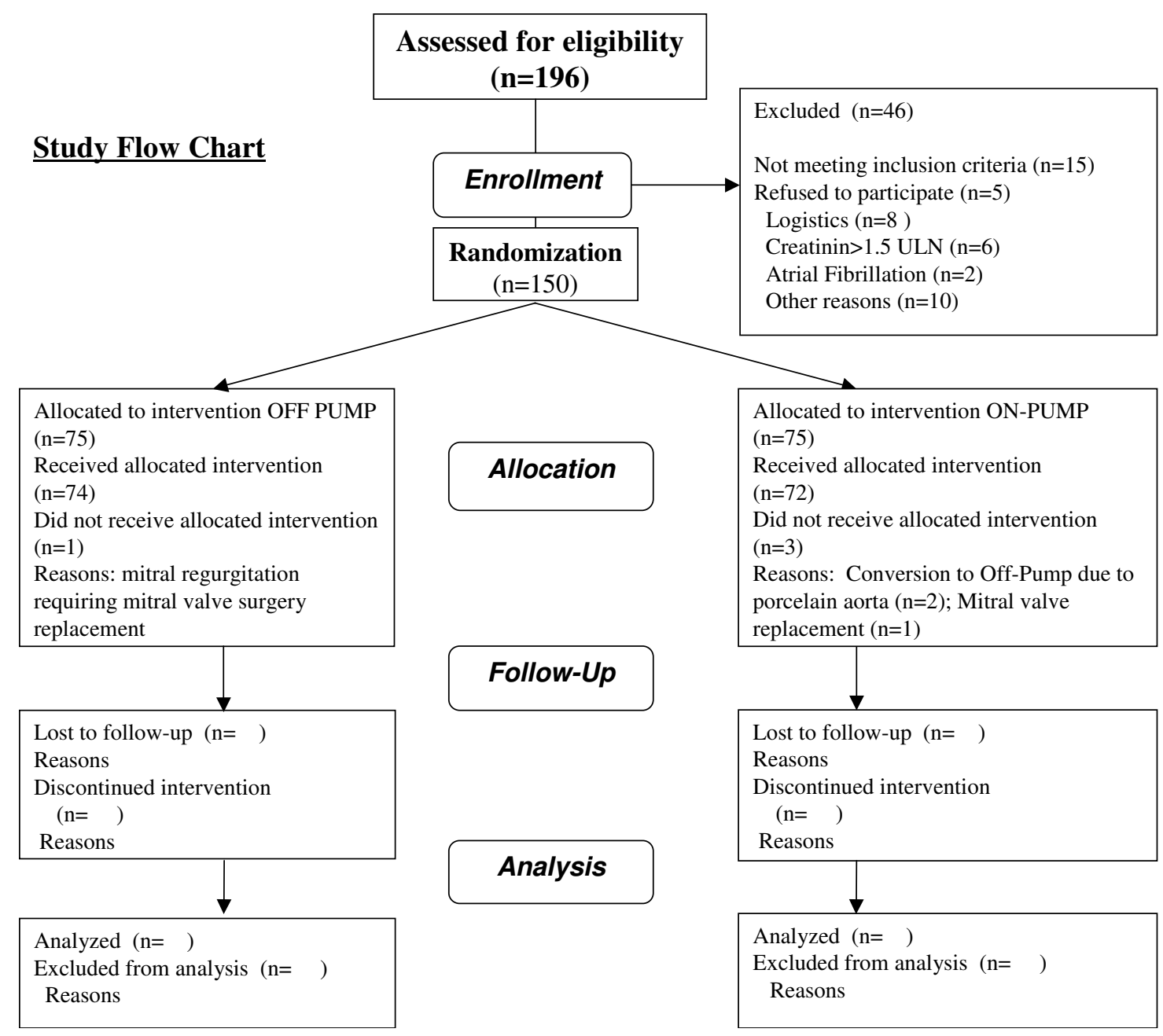

Figure I

Study Flow Chart. 
On-pump CABG uses a conventional roller pump cardiopulmonary bypass machine with an arterial filter, continuous ultra filtration, and a central temperature that is allowed to drift to $34^{\circ} \mathrm{C}$. Myocardial protection is provided by $34^{\circ} \mathrm{C}$ antegrade blood-potassium cardioplegia delivered every 25 minutes.

Off-pump coronary artery bypass grafting uses a deep pericardial stitch and vacuum stabilizers for exposure. Intra coronary shunts are systematically used.

The planned/performed distal anastomoses index and the reason for eventual incomplete revascularisation as well as target coronary artery quality (scale $1-4$ ) are noted in the two groups.

Post operative care is under the responsibility of the same intensive care team and follows the rules and protocols of the hospital ICU.

\subsection{Blinding}

Treatment group is blinded to patients, family and investigators responsible for the MDCT angiographic control, neuro-psychological tests and follow-up.

\subsection{Data Collection}

Demographic data, pre operative, intra operative, post operative and follow up variables are reported in a dedicated Case Report Form. Event adjudication is made by hospital physicians according to standardized definitions (Table 2). Protocol adherence and data collection reliability are monitored by an independent external body verifying conformity with good clinical practice requisites (EuroTrials, Scientific Consultants, Lisbon, Portugal).

\subsection{Assessment of results}

\section{al Principal end point}

The study main end point, comparison of graft patency at 4 to 6 weeks between the two groups, is performed by a GE Lightspeed 16 slice multidetector computed tomography using a standard protocol already described [23]. A cardiologist and a radiologist, with previous knowledge of the number and distribution of grafts performed but not the technique that was used assess the grafts using multiplane reformatted images, maximal intensity projection images and three dimensional reconstructions. Quality image is graded as good/excellent, minor artefacts, moderate artefacts and major artefacts. Only good/excellent images and images with minor artefacts are considered for analysis.

Each graft is independently graded using a three grade scale: occluded graft, graft stenosis of $50 \%$ or more, and stenosis $<50 \%$ or no stenosis at proximal anastomoses (if any), body graft and distal anastomosis [23].

Graft occlusion is defined as absence of contrast material along the course of the graft, through the graft anastomosis to the native coronary artery or to the following graft segment and native vessel. In sequential bypass grafts, each anastomosis of one graft is counted as a separate graft. The overall graft score is the worst of the three subcsores. Graft patency is evaluated according to graft material, site, as well as number of anastomoses patent per patient.

\section{b/ Secondary endpoints}

Secondary end points are:

Table 2: Definition of Post Operative Events

\begin{tabular}{|c|c|}
\hline Outcome & Definition \\
\hline Mortality & Autopsy result. \\
\hline Arrythmias & $\begin{array}{l}\text { Episodes requiring administration of iv anti arrythmics or electric choc. Atrio-ventricular conduction disturbances } \\
\text { defined as requiring temporary or definitive pace maker. }\end{array}$ \\
\hline Reoperation & Reoperation or recatheterisation for myocardial ischemia. \\
\hline Myocardial Infarction & New $Q$ wave or increase in CK-MB $>5 x U L N$.or troponin $T>$ or $=1 \mathrm{ng} / \mathrm{ml}$. \\
\hline CK-MB and troponin $\mathrm{T}$ & Mean values at 6,12 and 24 hours post operatively. \\
\hline Cardiac Faillure & Cardiac resuscitation manoeuvres or intra aortic balloon pump. Level of inotropic support. \\
\hline Neurologic Events & $\begin{array}{l}\text { Coma (Glasgow score), stroke (focal neurologic deficit }>24 \text { hours), transient ischemic attack (focal neurologic } \\
\text { deficit }<24 \text { hours), delirium. }\end{array}$ \\
\hline Respiratory Complications & $\begin{array}{l}\text { ARDS, ventilation }>24 \text { hours, pneumonia, thoracic effusion or pneumothorax requiring drainage and } \mathrm{PaO} 2 \text { at } \\
\text { discharge from ICU. }\end{array}$ \\
\hline Renal Complications & $\begin{array}{l}\text { Renal dialysis, ultra filtration, need for continuous iv diuretics, serum creatinin increase between base line and } \\
\text { ICU discharge. }\end{array}$ \\
\hline Bleeding Complications & Reoperation for bleeding, blood products administration, total blood drainage. \\
\hline Infectious Complications & Sternal infection (deep and superficial); septicaemia. \\
\hline Gastro Intestinal Complications & Cholecystitis, Pancreatitis, Mesenteric Ischaemia, GI perforation, GI bleeding, Intestinal Occlusion. \\
\hline Ressource utilisation & Readmision to ICU; ICU and hospital length of stay. \\
\hline
\end{tabular}


1- Revascularisation index: number of planned distal anastomoses/number of performed distal anastomoses.

2- Adverse events at hospital discharge and at 4-6 weeks (Table 2). Each complication is separately analysed, Major adverse events rate is also recorded as the cumulative incidence of mortality, myocardial infarction, stroke or TIA, dialysis, reoperation for ischemia, ventilation $>24 \mathrm{~h}$.

\section{3- Neuro-cognitive assessment}

Neurocognitive assessment consisted on the performance at baseline and at 4-6 weeks of the following tests:

- Digit span (from Wechsler Adult Intelligence Scale-III)

- Digit Symbol (from Wechsler Adult Intelligence Scale III)

- Grooved pegboard test

- Auditory verbal learning test

- Complex Figure Test

- Trail making test A and B

- Letter Word Fluency

- Controlled Oral Word Association (COWA)

- Hospital Anxiety Depression Scale (HADS)

- Beck Depression Inventory

The assessment of 50 healthy demographically matched control subjects, with the same test battery and test-re-test procedures, will enable the calculation of $\mathrm{T}$-scores for each neuropsychological evaluation. Decline will be defined by a drop equal or greater to 15 points.

4- Follow up at 6 months and 1 year will compare functional status, stress test, adverse events and quality of life by EuroQOL between the two groups.

\section{cl Tertiary end point}

is the analysis of costs: direct patient care related in hospital costs.

\subsection{Statistical analysis}

a/ Sample size

PROMISS was planned as a non inferiority study of one intervention (OPCAB) relative to the reference operation (on-pump). This study was designed with adequate power (80\%; $\alpha$ level of 0.05$)$ to detect an absolute difference in patency rates between groups in either direction as small as $5 \%$. The calculation resulted in a total of 426 units (distal graft anastomoses). Since each patient has by the inclusion criteria at least 3 grafts, 71 patients in each group are necessary ( 213 grafts) for a total of 142 patients. Considering the possibility of 4 patients' withdrawal in each group, a total sample of 150 patients was established.

\section{b/ Statistical analysis}

Discrete data are presented as numbers and percentages; continuous data are presented as median, mean (SD) and compared using t test or the Mann-Whitney test. Dichotomous morbidity and mortality outcomes are analyzed using the Fisher exact test. Ordered categorical outcomes are compared between groups using the Mantel Haenszel $\chi^{2}$.

Dichotomous patency outcomes or graft stenosis $>50 \%$ are analyzed using the Fisher exact test. Mean number of patent anstomosis per patient is compared using the $t$ test or the Mann-Whitney test.

\section{Discussion}

The Prospective Randomized Comparison of Off-Pump and On-Pump MultI-vessel Coronary Artery BypasS Surgery (PROMISS) was designed to try to answer the question of whether or not coronary artery bypass grafting performed off-pump has the same early graft patency as if performed on-pump in arrested hearts.

The sample size was calculated in order to detect $a+/-5 \%$ difference between the two techniques. This trial includes only patients with multi vessel disease with an indication to perform at least 3 distal coronary artery anastomoses for two reasons. Firstly to compare the two techniques in terms of completeness of revascularisation since various series have reported a lower number of grafts/patient in OPCAB relative to conventional on-pump surgery [8]. Secondly to compare OPCAB graft patency with the reference method in a high percentage of cases requiring revascularisation of the lateral wall for its reported increased technical difficulty $[24,25]$. Complete revascularisation is mandatory including the lateral wall since it is one reason for the superior outcomes of CABG compared with percutaneous coronary intervention $[26,27]$.

Few studies have compared OPCAB versus CABG with $\mathrm{CPB}$ among randomly assigned patients unselected for coronary anatomy, ventricular function or co-morbidities except renal insufficiency. All surgeries using standardized techniques were performed by a single surgeon experienced with both methods in an attempt to eliminate technical variability. 
This is the first randomized study comparing early graft patency in off-pump and on-pump coronary bypass surgery using 16 slice multidetector computed tomography in patients with multivessel disease. Although 64 slice multidetector computed tomography has improved temporal and spatial resolution, there is ample evidence that, with appropriate heart rate reduction, 16 slice MDCT is able to accurately detect graft occlusion and stenosis compared to conventional coronary angiography $[23,28,29]$.

Limitations of this study include the fact that physicians involved in patient care, except those assessing graft patency, are not blinded to treatment method, which can eventually bias treatment decision making and length of stay. Another limitation is the single surgeon type of study which may prevent generalizability of the results. Finally, the assumption made for sample size calculation that graft patency within a patient is an independent research unit may not always apply requiring application of statistical adjustment.

\section{Conclusion}

The Prospective Randomized Study of graft patency in Off-pump and On-pump MultI-Vessel coronary artery bypasS Surgery (PROMISS) using multidetector computed tomography, has the potential to shed new light on the ongoing debate regarding these two techniques and therefore may have implications on the revascularisation strategy.

\section{List of Abbreviations}

ARDS: Acute Respiratory Distress Syndrome; CABG: coronary artery bypass grafting; CAD: Coronary Artery Disease; ICU: Intensive Care Unit; MDCT: Multidetector Computed Tomography; OPCAB: Off-Pump Coronary Artery Bypass; PROMISS: Prospective Randomized Study of graft patency in Off-pump and On-pump MultI-Vessel coronary artery bypasS Surgery; TIA: Transient Ischemic Attack; ULN: Upper Limit of Normal of Laboratory value.

\section{Competing interests}

The authors declare that they have no competing interests.

\section{Authors' contributions}

All authors read and approved the final manuscript. Specifically, MSU principal investigator, conceived and designed the study and drafted the manuscript. FM is responsible for performing computed tomography studies and helped draft the manuscript. SC conceived and coordinated the neurocognitive evaluation and helped with statistical analysis. MPM helped draft the manuscript and supervised the research project.

\section{Acknowledgements}

Armindo Mesquita for his contribution in patients' follow-up.
Sofia Nunes da Silva, Joana Carvalho and Raquel Travassos are acknowledged for performing the neuropsychological tests, Pedro Aguiar for performing the statistical analysis Joao Bérrio for logistical and technical support on data collection and Carla Martinho and João Costa for secretarial assistance.

PROMISS benefited from the support of Sociedade de Gestão Hospitalar Cruz Vermelha Portuguesa, Lisbon, Portugal where the interventions and follow up studies took place and who funded the multidetector computed tomography procedures.

Merck Foundation, Lisbon, Portugal supported this work through EuroTrials Scientific Consultants, for data collection, monitoring and data analysis support.

None of the authors received direct funds for this study.

Neither Sociedade de Gestão Hospitalar - Cruz Vermelha Portuguesa nor Merck Foundation had any role in study design, manuscript draft or decision to submit the manuscript.

\section{References}

I. Yusuf S, Zucker D, Peduzzi P, Fisher LD, Kennedy JW, Davis K, Killip T, Passamani F, Norris R: Effect of coronary artery bypass surgery on survival: overview of 10 -year results from randomized trials from the Coronary Artery Bypass Surgery Trialists Collaboration. Lancet 1994, 344:563-570.

2. Ferguson TB, Hammill BG, Peterson ED: A decade of change. Risk profiles and outcomes for isolated coronary bypass grafting procedures, 1990-1999: A report from the STS National Database Committee and the Duke Clinical Research Institute. Ann Thorac Surg 2002, 73:480-490.

3. Buffolo E, Andrade JC, Branco JN, Aguiar LF, Ribeiro EE, Jatene AD: Myocardial revascularisation without extracorporeal circulation. Eur J Cardiothorac Surg 1990, 4:504-507.

4. Menasché $P$ : The systemic factor: the comparative roles of cardiopulmonary bypass and off-pump surgery in the genesis of patient injury during and following cardiac surgery. Ann Thorac Surg 200I, 72:S2260-2266.

5. Ascione R, Angelini GA: Off-pump coronary artery bypass surgery: the implications of the evidence. J Thorac Cardiovasc Surg 2003, I 25:779-78|.

6. Sedrakyan A, Albert WW, Parashar A, Bass EB, Treasure T: Offpump surgery is associated with reduced occurrence of stroke and other morbidity as compared with traditional coronary artery bypass grafting. A meta-analysis of systematically reviewed trials. Stroke 2006, 37:2759-2769.

7. Cheng DC, Bainbridge D, Martin JE, Novick RJ: Evidence based peri-operative clinical outcomes research group. Does offpump coronary artery bypass reduce mortality, morbidity and resource utilisation when compared with conventional coronary artery bypass? A meta-analysis of randomized trials. Anesthesiology 2005, 102:188-203.

8. Selke F, Di Maio M, Caplan LR, Ferguson B, Gardner TJ, Hiratzka LF, Isselbacher EM, Lytle BW, Mack MJ, Murkin JM, Robbins RC: Comparing on-pump and off-pump coronary artery bypass grafting. Numerous studies but few conclusions. A Scientific Statement from the American Heart Association Council with the Interdisciplinary Working Group on Quality of Care and Outcomes Research. Circulation 2005, I I I :2858-2864.

9. Sergeant $P$, Wouters P, Meyns B, Bert C, Van Hemelrijck J, Bogaerts $C$, Sergeant G, Slabbaert K: OPCAB versus early mortality and morbidity: an issue between clinical relevance and statistical significance. Eur J Cardiothorac Surg 2004, 25:779-785.

10. Bonchek L: Off-pump coronary bypass: Is it for everyone? J Thorac Cardiovasc Surg 2002, 1 24:43I-434.

II. Sabik JF, Blackstone EH, Lytle BW: A benchmark for evaluating innovative treatment of left main coronary disease. Circulation 2007, I I 6(II Suppl):1232-239.

12. Puskas JD, Williams WH, Mahoney EM, Huber PR, Block PC, Duke PG, Staples JR, Glas KE, Marshall JJ, Leimbach ME, McCall SA, Petersen 
RJ, Bailey DE, Weintraub WS, Guyton RA: Off-pump vs conventional coronary artery bypass grafting: early and I year graft patency, cost and quality of life. JAMA 2004, 29 I: I 84I-I849.

13. Kobayashi J, Tashiro T, Ochi M, Yaku H, Watanabe G, Satoh T, Tajusari O, Nakajima H, Kitamura S, Japanese Off-pump Coronary Revascularisation Investigation (JOCRI) Study Group: Early outcome of randomized comparison of off-pump and on-pump multiple arterial coronary revascularisation. Circulation 2005, I / 2(Suppl): | 338-1343.

14. Khan EN, De Souza A, Mister R, Flather M, Olague J, Davies S, Collins $P$, Wang D, Sigwart U, Pepper J: A randomized comparison of off-pump and on-pump multivessel coronary artery bypass surgery. N Engl J Med 2004, 350:2 I-8.

15. Kim KB, Lim C, Lee C, Chae I, Oh BH, Lee MM, Park YB: Off-pump coronary artery bypass may decrease the patency of saphenous vein grafts. Ann Thorac Surg 200I, 72:S1033-1037.

16. Bashore TM, Bates ER, Berger PB, Clark DA, Cusmo JT, Dehmer G], Kern MJ, Laskey WK, O'Laughlin MP, Oesterle S, Popma J], O'Rourke RA, Abrams J, Bates ER, Brodie BR, Douglas PS, Gregaretos G, Hlatky MA, Hochman JS, Kaul S, Tracy CM, Warters DD, Winter DD Jr: American College of Cardiology/Society for Cardiac Angiography and Interventions Clinical Expert Consensus Document on cardiac catheterisation laboratory standards. A report of the American College of Cardiology Task Force on Clinical Expert Consensus Documents. J Am Coll Cardiol 200I, 37:2170-22|4.

17. Nieman K, Pattynama PMT, Rensin BJ, van Geuns RJM, de Feyter PJ: Evaluation of patients after coronary artery bypass surgery: CT angiographic assessment of grafts and coronary arteries. Radiology 2003, 229:749-756.

18. Yoo Kj, Choi D, Choi BW, Lim SH, Chang BC: The comparison of the graft patency after coronary artery bypass grafting using coronary angiography and multi-slice computed tomography. Eur J Cardiothorac Surg 2003, 24:86-9I.

19. Schlosser T, Konorza T, Hunold P, Kuhl H, Schmermund A, Barkhausen J: Non-invasive visualization of coronary artery bypass grafts using 16 detector row computed tomography. J Am Coll Cardiol 2004, 44: I224-I 229.

20. Martuscelli E, Romagnoli A, D'Eliseo A, Tomassini M, Razzini C, Sperandio M, Simonetti G, Romeo F, Mehta JL, et al.: Evaluation of venous and arterial conduit patency by 16-slice spiral computed tomography. Circulation 2004, I I 0:3234-3238.

21. "Guideline for Good Clinical Practice", ICH Tripartite Guideline. 2002.

22. Moher D, Schulz KF, Altman DG: The CONSORT statement: revised recommendations for improving the quality of reports of parallel-group randomised trials. Lancet 200I, 357(9263): I|9|-I| 94.

23. Sousa Uva M, Matias F, Mesquita A, Rogério Costa , José Baú MD, Albino Pedro MD, Manuel Pedro Magalhães MD: Diagnostic Accuracy of 16-Detector Multi-Slice Computed Tomography for Graft Patency Evaluation After Coronary Artery Bypass Surgery. J Card Surg 2008, 23:17-22.

24. Cooley DA: Con: Beating heart surgery for coronary revascularisation: it is the most important development since the introduction of the heart-lung machine. Ann Thorac Surg 2000, 70: I779- I78I

25. Sabik JF, Gillinov AM, Blackstone EH, Vacha DC, Houghtaling PL, Navia J, Smedira NG, McCarthy PM, Cosgrove DM, Lytle BW: Does off-pump coronary surgery reduce morbidity and mortality? J Thorac Cardiovasc Surg 2002, 1 24:698-707.

26. Kleisli T, Cheng W, Jacobs MJ, Mirocha J, DeRobertis MA, Kass RM, Blanche C, Fontana GP, Raissi SS, Magliato KE, Trento A: In the current era, complete revascularisation improves survival after coronary artery bypass surgery. J Thorac Cardiovasc Surg 2005, 129:1283-1291.

27. Hannan E, Racz M, Holmes DR, King SB 3d, Walford G, Ambrose JA, Sharmo S, Katz S, Clark LT, Jones RH: Impact of completeness of percutaneous coronary intervention revascularisation on long-term outcomes in the stent era. Circulation 2006, I I3:2406-24I2.

28. Yamamoto M, Kimura F, Niinami H, Suda Y, Ueno E, Takeuchi Y: Non invasive assessment of off-pump coronary artery bypass surgery by 16-channel multidetector-row computed tomography. Ann Thorac Surg 2006, 81 :820-827.
29. Schroeder S, Achenbach S, Bengel F, Burgstahler C, Cadermartiri F, de Feyter P, George R, Kaufmann P, Kopp AF, Knuuti J, Ropers D, Schuijf J, Tops LF, Bax JJ: Cardiac computed tomography: indications, applications, limitations and training requirements. Report of a Writing Group deployed by the Working Group Nuclear Cardiology and Cardiac CT of the European Society of Cardiology and the European Council of Nuclear Cardiology. Eur Heart J 2008, 29:53 I-556.
Publish with Bio Med Central and every scientist can read your work free of charge

"BioMed Central will be the most significant development for disseminating the results of biomedical research in our lifetime. "

Sir Paul Nurse, Cancer Research UK

Your research papers will be:

- available free of charge to the entire biomedical community

- peer reviewed and published immediately upon acceptance

- cited in PubMed and archived on PubMed Central

- yours - you keep the copyright 